\title{
Energization of ions in near-Earth current sheet disruptions
}

\author{
A. Taktakishvili \\ Abastumani Astrophysical Laboratory, Tibilisi, Republic of Georgia \\ R.E. Lopez and C.C. Goodrich \\ Department of Astronomy, University of Maryland, College Park
}

\begin{abstract}
In this study we examine observations made by AMPTE/CCE of energetic ion bursts during seven substorm periods when the satellite was located near the neutral sheet, and CCE observed the disruption cross-tail curren' in situ. We compare ion observations to analytic calculations of particle acceleration. We find that the acceleration region size, which we assume to be essentially the current disruption region, to be on the order of $1 R_{E}$. Events exhibiting weak acceleration had either relatively small acceleration regions (apparently associated with pseudobreakup activity on the ground) or relatively small changes in the local magnetic field (suggesting that the magnitude of the local current disruption was limited). These results add additional support for the view that the particle bursts observed during turbulent current sheet disruptions are due to inductive acceleration of ions.
\end{abstract}

\section{Introduction}

Energetic ( $>10 \mathrm{keV}$ ) particle bursts in the Earth's magnetotail have been discussed by a number of authors [e.g., Krimigis and Sarris, 1980]. The observations discussed in those papers were made by the IMP series of spacecraft, which sampled the more distant ( $30 R_{E}$ to $40 R_{E}$ ) magnetotail. These particle bursts often have maximum energies of 1-2 Mev, in spite of the fact that the potential drop across the magnetotail is on the order of $100 \mathrm{keV}$. Thus the generation of such particles must be related to processes such as those thought to operate during a substorm. The bursts are generally substormassociated and it has been suggested that they are related to the generation of reconnection regions in the tail [Hones, 1984], or acceleration of particles through inductive electric fields [Krimigis and Sarris, 1980]. Calculations based on this idea have shown that acceleration up to $\mathrm{MeV}$ energies is possible [Galeev, 1979; Taktakishvili and Zelenyi, 1990] and that the calculated spectrum and velocity dispersion of the accelerated particles is consistent with observations [Zelenyi et al., 1990; Taktakishvili et al. 1993]. Pellinen and Heikkila [1978] also discussed inductive acceleration of particles in the magnetotail, although they attributed much of the energization to betatron acceleration as the particles drifted into regions of high magnetic field.

Recently there have been detailed studies of substorm phenomena in the inner $\left(R<10 R_{E}\right.$ ) magnetotail using data from the AMPTE/CCE satellite [e.g., Lopez et al., 1993 and references therein]. These studies have highlighted the

\section{Copyright 1995 by the American Geophysical Union.}

Paper number 94GL03384

0094-8534/95/94GL-03384\$03.00 importance of the inner magnetotail and provided considerable evidence that substorms are initiated in this region [Lopez et al., 1990; Lopez, 1992; Lui et al., 1992]. Moreover, there are several examples of substorms when CCE was located within the current disruption region itself in close proximity to the neutral sheet [e.g, Lopez et al., 1989; Lui et al., 1992].

During these current sheet disruption events, bursts of energetic particles were observed in conjunction with strong, turbulent magnetic field perturbations, leading to the suggestion that the particles in these bursts might have been accelerated by inductive electric fields within the disruption region [Lopez et al., 1989]. Support for near-Earth acceleration of ions by current sheet disruption has also been presented by Reeves et al. [1992]. In fact it has long been recognized that the dispersionless injections regularly seen at geosynchronous orbit are likely due to some near-Earth acceleration process, otherwise energy-dependent drifts would disperse them [e.g., Baker, 1984].

In the following sections we will present observations of current sheet disruption and evidence for particle energization. Those observations will allow us to calculate the maximum energy of the accelerated particles as a function of the $X$ extent of the acceleration region using the model of Zelenyi et al. [1990]. Comparing this simple model of acceleration with the energetic particle observations we will find surprisingly good agreement with expected acceleration region source sizes.

\section{Observations}

The data to be examined in this paper consist of energetic particle and magnetic field measurements from AMPTE/CCE. CCE is in an equatorial elliptical orbit with apogee at $8.8 R_{E}$. It has a spin-rate of 6 seconds and a spin axis roughly parallel to the Earth-Sun line. Energetic particle measurements were made by the Medium Energy Particle Analyzer (MEPA). MEPA collects data using two detectors, the Time-Of-Flight (TOF) head and the ion head. We will only present all-ion data collected by the ion head since the all-ion channels from the TOF head are not directly compatible with the ion head channels. MEPA experienced a steadily increasing gain shift and the energy boundaries of the channels have been individually adjusted accordingly. We also present data from the magnetic field experiment.

Figure 1 presents magnetic field data from a sample event that occurred on October 19, 1986, just before 2212 UT when CCE was at a radial distance of $7.7 R_{E}$ and at 23.1 MLT. This event is one of those discussed by Lui et al. [1992]. The data are presented in $V D H$ coordinates, where $H$ is along the dipole axis, $V$ is radially outward, and $D$ is positive eastward. Prior to the onset, the magnetic field value was about $28 \mathrm{nT}$, well below 

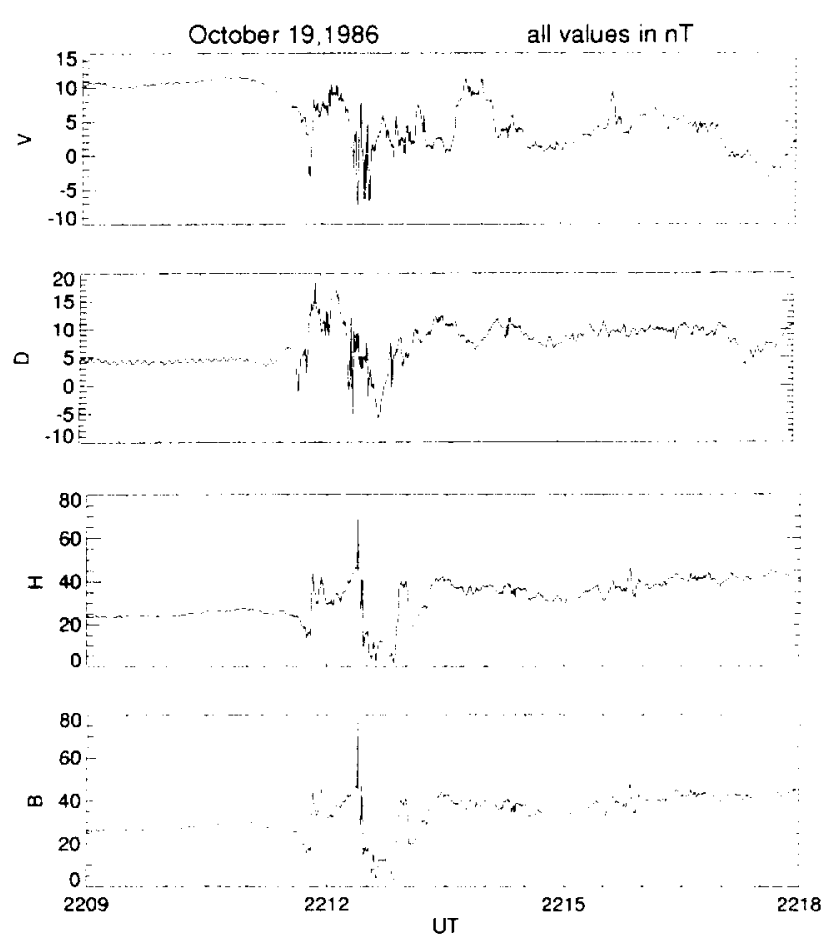

Figure 1. Magnetic field data from CCE on October 19, 1986. The data are in $V D H$ coordinates (see text) and have a resolution of $0.6 \mathrm{~s}$.

the dipole field value of $67 \mathrm{nT}$, and the $V$ component was small and positive, indicating that the satellite was located near the tail midplane just south of the center of the current sheet (the 1$2 \mathrm{nT}$ variations are spin noise due to a small offset between the magnetometer booms). At the onset of the event the magnetic field exhibited a turbulent reconfiguration toward a more dipolar field value over a 1.5 -minute interval. The final field magnitude was about $45 \mathrm{nT}$, indicating that the local cross-tail current had been disrupted.

Spin-averaged, ion-head energetic particle data with $24 \mathrm{~s}$ resolution are presented in Figure 2 . The gaps in the plots are periods when the TOF head collected all-ion data. At 2212 UT, there was a sharp increase in the energetic ion flux. The increase extended only into the $328-620 \mathrm{keV}$ channel, and we take the center point of that channel to be the maximum energy in the injection. Lower energy channels (not shown) also recorded the injection, which was essentially dispersionless over a wide range of energy. This indicates that the ions must have been energized nearby, or energy dependent drifts would have quickly dispersed them [e.g., Reeves et al., 1992].

The other six events in this study are also taken from Lui et al. [1992]. We only take those events for which it was possible to obtain the parallel and perpendicular components of the plasma pressure prior to the disruption; these are summarized in Table 3 of Lui et al. [1993]. Using those values of the pressures it is possible to obtain the asymptotic lobe field strength from the local observations [Lui, 1993], which is needed to calculate the maximum energy. Each of these events showed behavior similar to that illustrated in our sample event. They are marked by a turbulent reconfiguration of the magnetic field such as seen in Figure 1, and there was a maximum energy in the injection. In the following section we compare these observations to results obtained using the particle acceleration model of Zelenyi et al. ]1990].

\section{Comparison Between Observations and Model}

The calculations of Zelenyi et al. [1990] and Taktakishvili and Zelenyi [1990] have been successful in providing a framework for understanding the characteristics of magnetotail energetic particle bursts observed by the IMP series of spacecraft. Can these calculations provide some frame of reference for understanding near-Earth particle acceleration as well? Although Zelenyi et al. [1990] assumed a neutral line to be the agent of the acceleration, the calculation requires only a specified inductive electric field and it is not strictly dependent upon the physical origin of that electric field. In fact, CCE observations are more consistent with a turbulent breakup and disruption of the current sheet than with a near-Earth neutral line, but even in this case a large inductive electric field should be present [Lopez et al., 1989]. Another concern is the applicability of the model geometry to the near-Earth regime. The model was formulated for the distant tail and it does not contain the large $X$-gradients found in the near-Earth region. However, a sheet-like geometry is not a bad approximation to the late growth phase at $9 R_{E}$ where the magnetic field can become quite tail-like. Therefore, although we recognized the need for more realistic calculations, we feel that the model can provide some insight into the observations.

Zelenyi et al. [1990] considered the inductive electric field produced by an explosive magnetic perturbation associated with the development of a tearing mode instability to be the agent of particle energization. The magnetic configuration is given by a superposition of an equilibrium Harris field and the perturbed normal magnetic field, so that $B=B_{0 x} \tanh (z / L)+$ $B_{z}(t) \sin (k x)$, where $B_{0 x}$ is the value of the lobe field and $k$ is the tearing-mode wave number corresponding to the length of a
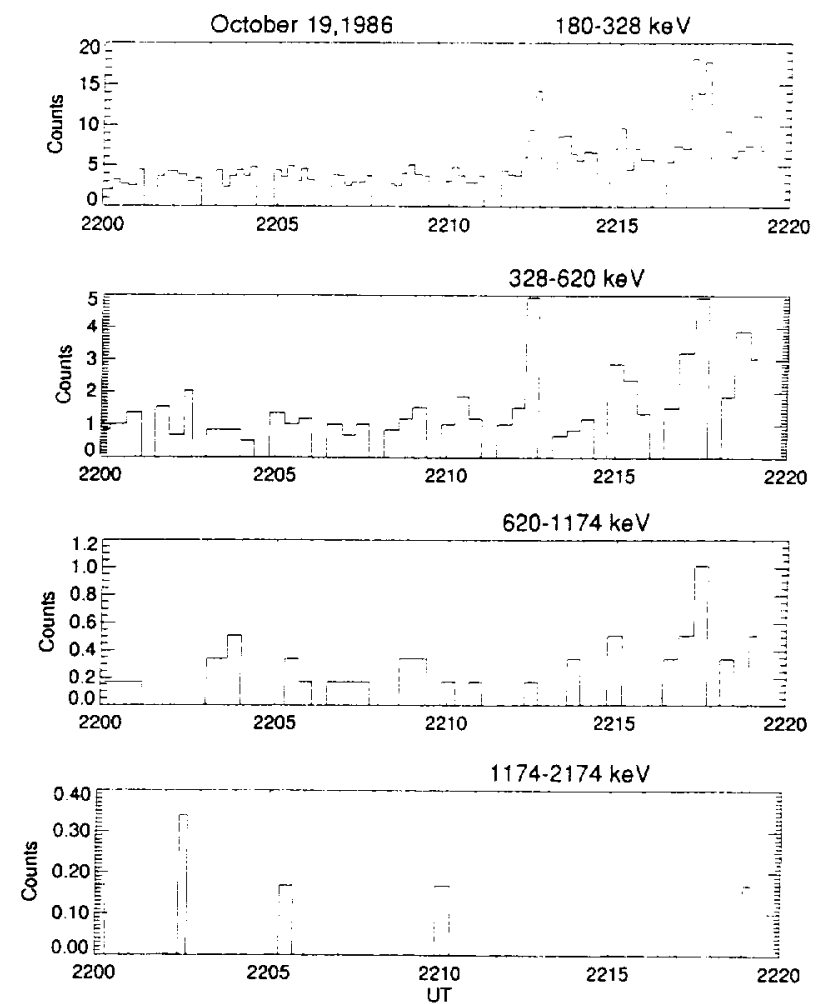

Figure 2. Spin-averaged ion counts/s for the October 19, 1986 event. The time resolution of the data is either $12 \mathrm{~s}$ or $24 \mathrm{~s}$, and there was a clear maximum energy in the injection. 


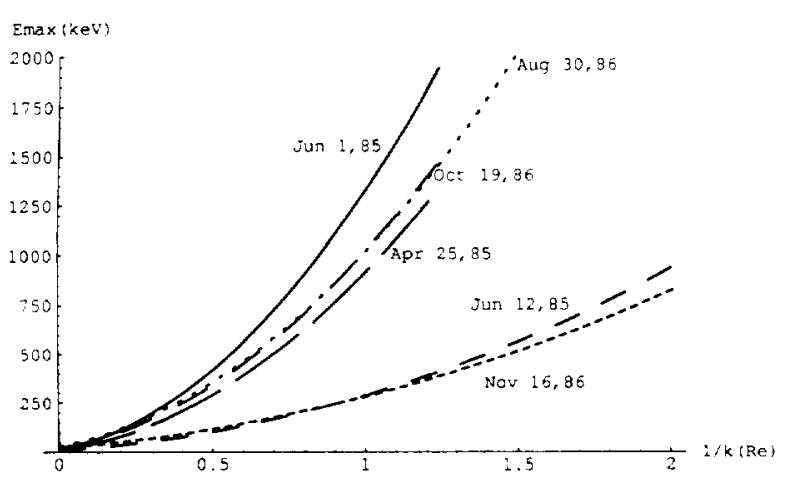

Figure 3. Maximum energy of the accelerated ions as a function of the acceleration region size in the $\mathrm{X}$ direction as derived from the model of Zelenyi et al. [1990].

magnetic island; it is assumed to be one dimensional along the $X$-axis and $1 / \mathrm{k}$ corresponds to the size of the acceleration region. The normal perturbation is given as $B_{z}(t)=B_{0 z} /(1-t / \tau)$, where $\tau$ is the characteristic time of the explosive growth [Galeev, 1979]. It is assumed that there is a saturation time, $t_{s}$ $<\tau$, when $B_{z}$ reaches its final value (after which it is constant in time), and that the linear stage of the tearing mode development may be neglected, since it does not produce significant acceleration. The equations of motion for particles within the acceleration region (that region near to the neutral line) are solved analytically [e.g., Zelenyi et al., 1990]. Particles that remain within the acceleration region up to the saturation time, $t_{3}$, reach the maximum energy, which is given by $E_{0}\left[1+\omega_{c} B_{0 z}\left(B_{z}\left(t_{s}\right) / B_{0 z}-1\right) / B_{0 x} k V_{T}\right]^{2}$, where $E_{0}$ is the initial energy, $\omega_{c}$ is the cyclotron frequency in the lobe field, and $V_{T}$ is the initial thermal velocity of the particles. However, particles may be thrown out of the acceleration region before they reach the maximum energy by the non-zero Lorentz force resulting from the normal component of the field.

The maximum energy depends on the initial temperature, initial and final $B_{z}$, the lobe field strength, and the $X$ extent of the acceleration region (essentially $1 / \mathrm{k}$ ). For our seven events we are able to directly observe or derive all of these quantities with the exception of the size of the acceleration region. Figure 3 shows the maximum expected energy calculated as a function of acceleration region size for the seven events. Most of the curves are clustered together, with the exception of the June 12, 1985 and November 16, 1986 events. These events had relatively low final magnetic field values relative to the pre-onset lobe magnetic field. Table 1 lists the seven events, the magnetic parameters used in the calculations, and the observed maximum energy in the burst (the center point of the highest-energy channel that showed evidence of the burst). The final column shows the inferred size in the $X$ direction of the acceleration region and (in parenthesis) the range of acceleration region size derived from the bandwidth of the maximum energy channel. Five of the events have acceleration region sizes (which we roughly equate to the size of the current disruption region) of 1 to $1.5 R_{E}$ whereas two events (April 25, 1985 and October 19, 1986) have acceleration region sizes of $0.7 R_{E}$ and $0.6 R_{E}$, respectively.

Does this result have any relationship to reality? In fact there is reason to suspect that the two events with a small acceleration region size were limited to a small radial extent in the magnetotail. One of the two weak events (April 25, 1985) has been the focus of a previous extensive study [Lopez et al., 1990]. Notably, although the local time extent of the substorm activity was great, there was almost no poleward expansion of activity and the substorm was quite small in terms of AE, local auroral zone negative bays, and mid latitude perturbations. Such behavior seems to be a typical feature of pseudobreakups or weak substorms [Koskinen et al., 1992; Ohtani et al., 1993]. We have also examined the data from the EISCAT magnetometer cross (not presented here) for the October 19, 1986 event. Those data show a similar story: the electrojet was relatively weak $\left(\Delta \mathrm{H}_{\max } \approx-140 \mathrm{nT}\right)$ and confined to low latitudes with essentially no poleward expansion around the period of current disruption displayed in Figure 1. In contrast, four of the five remaining events show clear evidence of poleward expansion of activity. In the case of the fifth event, November 16,1986, it is difficult to say because the station nearest to the CCE meridian that recorded substorm activity was Churchill, 1.3 hours to the east and at a relatively high magnetic latitude. Given the close connection between ionospheric and near-Earth magnetotail activity at the onset of a substorm [e.g., Lopez et al., 1993], latitudinally limited ionospheric activity would would suggest a radially limited region of activity in the near-Earth magnetotail.

Table 1. Current disruption events and acceleration region sizes in the $X$ direction

\begin{tabular}{ccccccc} 
Date & UT & $\begin{array}{c}\mathrm{R} \text { in } \\
R_{E}\end{array}$ & MLT in hours & $\begin{array}{c}\text { Magnetic Field (nT) } \\
\text { initial } \mathrm{B}_{\mathrm{Z}}, \text { final } \mathrm{B}_{Z}, \\
\text { lobe (inferred) }\end{array}$ & $\begin{array}{c}\text { Max. Energy } \\
\text { (channel } \\
\text { center) }\end{array}$ & $\begin{array}{c}\text { Acceleration } \\
\text { region size in } R_{E} \\
\text { (min, max) }\end{array}$ \\
\hline April 25, 1985 & 0112 & 8.6 & 1.4 & $17.1,35,55$ & $560 \mathrm{keV}$ & $0.7(0.6,0.8)$ \\
June 1, 1985 & 2121 & 8.6 & 23.7 & $8.1,30,56.6$ & $1453 \mathrm{keV}$ & $1.0(0.9,1.1)$ \\
June 12, 1985 & 0950 & 8.8 & 23.3 & $13.3,23,51.7$ & $301 \mathrm{keV}$ & $1.0(0.8,1.2)$ \\
August 28, 1986 & 1200 & 8.1 & 23.2 & $22.1,40,66.1$ & $936 \mathrm{keV}$ & $1.0(0.8,1.1)$ \\
August 30, 1986 & 1223 & 8. & 23.9 & $30.3,48,64.2$ & $1792 \mathrm{keV}$ & $1.4(1.2,1.5)$ \\
October 19, 1986 & 2212 & 7.7 & 23.1 & $26.5,45,61.9$ & $451 \mathrm{keV}$ & $0.6(0.5,0.7)$ \\
November 16, 1986 & 0447 & 8.8 & 20.7 & $13.9,22,58.1$ & $505 \mathrm{keV}$ & $1.5(1.2,1.7)$ \\
\hline
\end{tabular}


Two other events with weak acceleration (June 12, 1985 and November 16,1986 ) had relatively small changes in the local magnetic field, but larger acceleration region sizes. This situation suggests that local current disruption that is limited in magnitude, though not necessarily in size, also produces weak acceleration. This raises the question of what are the limiting factors on the disruption of the current and acceleration of particles. Koskinen et al. [1993] suggested that both the ionosphere and plasma sheet play a role. Similarly, Ohtani et al. [1993] pointed out the importance of ionospheric conductivity regarding the net current flow out of a disruption region in the magnetotail, and the radially limited current disruption region observed during a pseudobreakup. They suggest that the observed pseudobreakup was associated with weak auroral electron precipitation, and thus ionospheric conductivity. One speculation that we would like to put forward is that in situations where the ionospheric conductivity severely restricts the growth of a current disruption region, one obtains the phenomena associated with a pseudobreakup, i.e., limited latitudinal expansion, a radially limited current disruption region, and weak particle acceleration in the magnetotail. On the other hand, if plasma sheet parameters result in a low saturation level of the instability producing the disruption, but the ionosphere is fully receptive to the current diverted through it, there may be considerable spatial expansion of ionospheric and magnetotail activity, but limited particle acceleration in the magnetotail.

Despite the crudeness of the analytic model, the reasonable correspondence with the observations is encouraging, and it supports the contention that particle acceleration by inductive electric fields in current sheet disruption regions is a valid perspective for understanding near-Earth particle acceleration during substorms. It also provides a possible framework for understanding weak particle acceleration in terms of either limiting ionospheric or magnetotail conditions. In order to investigate these issues further, and determine whether the speculation introduced above has merit, investigations of Geotail, ISEE and IRM data should be conducted.

\section{Conclusion}

We have examined observations made by AMPTE/CCE of energetic ion bursts during seven substorm periods when $\mathrm{CCE}$ was located near the neutral sheet and observed the disruption of the cross-tail current in situ. We have compared our observations to analytic calculations of particle acceleration as discussed by Zelenyi et al. [1990], using local observations to provide all parameters for calculating the maximum energy as a function of the acceleration region size in the $X$ direction. Our results indicate that this simple analytic formalism provides a reasonable prediction of the maximum acceleration given typical acceleration region sizes. Moreover, we find that weak acceleration may be associated either with a limitation in the magnitude of the local current disruption, or with a limitation in the size of the disruption region. This latter phenomena may be associated with psuedobreakups. These results add additional evidence to the view that the particle bursts observed during turbulent current sheet disruptions are due to inductive acceleration of ions.

Acknowledgments The authors would like to thank A. T. Y. Lui , L. M. Zelenyi, and the Referees for helpful discussions and commeents, R. W. McEntire for providing the MEPA data, and T. A. Potemra for providing the magnetometer data. This work was supported by NASA contract NAS5-31208, by a grant from the NRC/CAST program, and by the IBM Department Grant made to the University of Maryland Advanced Visualization Laboratory.

\section{References}

Baker, D. N., Particle and field signatures of substorms in the near magnetotail, in Magnetic Reconnection in Space and Laboratory Plasmas, edited by E. W. Hones, Jr., 193-202, AGU, Washington, D. C., 1984.

Galeev, A. A., Reconnection in the magnetotail, Space Sci. Rev., 23, 411-425, 1979.

Hones, E. W., Jr., Plasma sheet behavior during substorms, in Magnetic Reconnection in Space and Laboratory Plasmas, edited by E. W. Hones, Jr., 178-184, AGU, Washington, D. C., 1984.

Krimigis, S. M., and E. T. Sarris, Energetic particle bursts in the Earth's magnetotail, in Dynamics of the Magnetosphere, edited by S.-I. Akasofu, 599-630, D. Reidel, Hingham, Mass., 1980.

Koskinen, H. E. J., R. E. Lopez, R. J. Pellinen, T. I. Pulkkinen, D. N. Baker, and T. Bosinger, Pseudobreakup and substorm growth phase in the ionosphere and magnetosphere, J. Geophys. Res., 97, 5801-5813, 1993.

Lopez, R. E, On the relative importance of magnetospheric and ionospheric processes during substorm breakup and expansion: A case study, in Proc. International Conference on Substorms, ESA, SP$335,425-428,1992$.

Lopez, R. E., A. T. Y. Lui, D. G. Sibeck, K. Takahashi, R. W. McEntire, L. J. Zanetti, and S. M. Krimigis, On the relationship between the energetic particle morphology and the change in the magnetic field magnitude during substorms, J. Geophys. Res., 94, 17105-17119, 1989.

Lopez, R. E., H. Luihr, B. J. Anderson, P. T. Newell, and R. W. McEntire, Multipoint observations of a small substorm, J. Geophys. Res., 95, 18897-18912, 1990.

Lopez, R. E., H. E. J. Koskinen, T. I. Pulkkinen, T. Bösinger, T. A. Potemra, and R. W. McEntire, Simultaneous observation of the poleward expansion of substorm electrojet activity and the tailward expansion of current sheet disruption in the near-Earth magnetotail, $J$. Geophys. Res., 98, 9285-9295, 1993.

Lui, A. T. Y., Inferring global characteristics of current sheet from local measurements. J. Geophys. Res., 98, 13423, 1993.

Lui, A. T. Y., R. E. Lopez, B. J. Anderson. K. Takahashi, L. J. Zanetti, R. W. McEntire, T. A. Potemra, D. M. Klumpar, E. M. Greene, and R Strangeway, Current disruptions in the near-Earth neutral shee region, J. Geophys. Res., 97, 1461-1480, 1992.

Ohtani, S., B. J. Anderson, D. G. Sibeck, P. T. Newell, K. Takahashi, L. J. Zanetti, T. A. Potemra, R. E. Lopez, V. Angelopoulos, R. Nakamura, D. M. Klumpar, and C. T. Russell, A multisatellite study of a pseudosubstorm onset in the near-Earth magnetotail, $J$. Geophys. Res., 98, 19355-19367, 1993.

Pellinen R. J., and W. J. Heikkila, Energization of charged particles to high energies by an induced substorm electric field within the magnetotail, J. Geophys. Res., 83, 1544-1550, 1978.

Reeves, G. D., G. Kettmann, T. A. Fritz, and R. D. Belian, Further investigation of the CDAW 7 substorm using geosynchronous particle data: Multiple injections and their implications, J. Geophys. Res., 97, 6417-6428, 1992.

Taktakishvili, A. L., and L. M. Zelenyi, Temporal dispersion structure of the proton bursts in the Earth's magnetotail, in Plasma Astrophysics, ESA SP-311, ed. by T. D. Guyenne, pp. 51-56, 1990.

Taktakishvili, A. L., L. M. Zelenyi, E. T. Sarris, Lopez, R. E., and D. V. Sarafopoulous, Temporal dispersion structures of proton and electron bursts in the Earth's magnetotail, Planet. Space Sci., 41, no. 6, 461467, 1993.

Zelenyi, L. M., J. G. Lominadze, and A. L. Taktakishvili, Generation of energetic proton and electron bursts in planetary magnetotails, $J$. Geophys. Res., 95, 3883-3891, 1990.

C. C. Goodrich and R. E. Lopez, Department of Astronomy, University of Maryland, College Park, MD 20742. (e-mail Internet ccg@avl.umd.edu, lopez@avl.umd.edu,)

A. Taktakishvili, Space Research Insitute, Russian Academy of Sciences, Profsoyuznaya 84/32, 117810 Moscow, Russia. (email,: Internet DZOGIN\%ESOC1.BITNET@vm.gmd.de)

(Received February 18, 1994; revised July 21, 1994 ; accepted September 15, 1994.) 